\title{
TESTS CONCERNING TUBERCLE BACILLI IN THE CIRCULATING BLOOD
}

\author{
E. C. SCHROEDER AND W. E. COTTON \\ BETHESDA, MD.
}

On Dec. 10, 1908, Dr. Randle C. Rosenberger of the Jefferson Medical College, Philadelphia, Pa., read a paper before the Philadelphia Pathological Society on the occurrence of tubercle bacilli in the circulating blood. The paper was afterward published in The American Journal of Medical Sciences for February, 1909. In this paper he recorded that he had made microscopic examinations of the blood of 125 tuberculous individuals, some of whom were affected with only incipient tuberculosis, and that he had found tubercle bacilli in the blood of every one of these individuals. In some cases only a few bacilli were seen, bur, to use his own words, "they were mostly in large numbers and clumps of 30 to 40 bacilli were not unusual, especially in cases of acute miliary tuberculosis." From his observations he formulated the conclusion, "It appears that tuberculosis in all its forms is a bacteriemia."

The occurrence of isolated tuberculous lesions in the bodies of otherwise tuberculous as well as otherwise healthy individuals, located in regions remote from the various channels that communicate with the exterior, gives the belief that tubercle bacilli occasionally float in the blood stream the character of a fact that is hardly open to question. The same is true when we consider cases of more or less generalized tuberculosis with many lesions in widely separated portions of the body, and cases of miliary tuberculosis with innumerable lesions of approximately, if not precisely, the same age and stage of development. But such occasional presence of tubercle bacilli in the circulating blood is a very different condition from their constant occurrence in it in sufficient numbers to justify the classification of tuberculosis as a bacteriemia. Hence, Rosenberger's conclusion was received with considerable surprise and decided incredulity.

Though the conclusion seems incredible, because it seems nearly impossible that a constant occurrence of tubercle bacilli in the blood of all tuberculous individuals could have been overlooked by the host of investigators who have studied tuberculosis with technic not greatly different from the one he used, we did not feel entitled to protest against it without offering some specific evidence to substantiate our protest. 
Rosenberger stated that he found tubercle bacilli on microscopic examination in the blood of every one of the 125 cases of tuberculosis he studied, notwithstanding that some of the cases were incipient and failed to show tubercle bacilli in the sputum. It was, therefore, almosit taken for granted that the microscopic examination of blood, according to his method, of animals affected with advanced and long standing tuberculosis and animals that were expelling tubercle bacilli from their bodies in large numbers, would reveal at least a few tubercle bacilli. A considerable number of such microscopic examinations were made, but not a tubercle bacillus was found in our blood preparations, and we have to record wholly negative results with the blood of tuberculous animals similar to the negative results obtained with the blood of tuberculous persons in two large New York hospitals. ${ }^{1}$

It is not uncommon for virulent tubercle bacilli to be present in animal substances in numbers too small to serve for their detection by optical methods. At the experiment station of the Bureau of Animal Industry, for example, we found the intra-abdominal injection of guineapigs -with suspected milk to be a test for tubercle bacilli that has fully 50 times the delicacy of a microscopic examination. Furthermore, tinctorial and optical methods of distinguishing between tubercle bacilli and other acid-fast bacteria are not wholly satisfactory; hence we decided to inject a sufficient number of guinea-pigs with blood from a sufficient number of certainly tuberculous cattle to show conclusively either that tubercle bacilli are commonly present in such blood or that they are not commonly present in such blood.

Incidentally we wish to say that Dr. Rosenberger's work is very weak in the extent to which he confirmed, by animal experiments, his surprising microscopic observations, which would be of the greatest value if true alone for the early and certain diagnosis of tuberculosis. In all he inoculated only two guinea-pigs of which he gives records-one with blood from a tuberculous person who was expelling tubercle bacilli per sectum and one with blood from a patient with acute miliary tuberculosis.

The development of tuberculosis in the latter guinea-pig can not be regarded as a remarkable phenomenon; there is nothing about the fact that a guinea-pig contracted tuberculosis after an injection of blood obtained from a case of acute miliary tuberculosis that necessitates a modification of our currently accepted views on the presence of tubercle bacilli in the circulating blood. That is to say, we need not look on tuberculosis as a bacteriemia because tubercle bacilli were demonstrated

1. Editorial in Med. Rec., New York, 1909, lxxr. 568. 
in blood, in which we have long taken their occurrence as a matter of course.

This leaves one guinea-pig that may have some evidential value, but we must not lose sight of the fact that it was injected with blood obtained from a person who was expelling tubercle bacilli from his body, and, hence, to some extent infecting his environment. We rnust also bear in mind that guinea-pigs are highly susceptible to tubercle bacilli injected into their bodies and that it is often impossible for an investigator who handles much tuberculous material, who is in frequent contact with tuberculous persons and whose environment may be characterized as containing tubercle bacilli, to eliminate all danger of extraneous tuberculous infection sufficiently to make a test satisfactory when he seeks to verify the tuberculous character of some material from a tuberculous individual by the injection of one, and only one, guinea-pig.

Our own tests, which follow, were made entirely with the blood of tuberculous cattle. In every case the blood was drawn from the jugular rein of the tuberculous animal and injected in its fresh, naturally warm state into the peritoneal cavity of a guinea-pig. The tuberculous cattle, as their records show, may be divided into four distinct lots according to the knowledge we have of their tuberculous condition.

Lot 1.-Four cattle, the precise tuberculous condition of which is known because they were killed and examined postmortem shortly after the blood was drawn from them for guinea-pig injections.

Lot 2.- Six cattle, known to be tuberculous because they had reacted with tuberculin, because tubercle bacilli were found in their feces on microscopic examination and because their feces were proven to be infectious by animal experiments.

Lot 3.-Nineteen cattle, known to be tuberculous because they had reacted with tuberculin and because tubercle bacilli were found in their feces on microscopic examination.

Lot 4.-Thirteen cattle, known to be tuberculous because they had reacted with tuberculin.

We made no attempt to treat the blood used for the injections in any way, because we assumed that the best results would be obtained with it by transferring it as rapidly as possible from the tuberculous cattle to the peritoneal cavities of the guinea-pigs. It was learned from the injections that guinea-pigs tolerate a relatively large quantity of bovine blood in their peritoneal cavity. The guinea-pigs that died shortly after and as the result of the blood injections, about 15 per cent. of all injected, with few exceptions showed extreme impaction and some 
inflammation of the large bowel, associated in several instances with invagination of the colon.

The possibility exists that the intraperitoneal injection of from 3 to 5 c.c. of fresh, warm blood from tuberculous cattle induces an immunity in guinea-pigs to the tubercle bacilli the blood may contain. Though this view is purely hypothetical and we know nothing to sustain it, we have undertaken an investigation to prove or disprove it, and will append the results to this article if they are ready before it goes to the printer.

The total number of cattle from which blood injections were made is 42 , and these, as their records show, represent a considerable variety relative to the severity and extent of the tuberculous disease with which they are affected, from animals that would not have been suspected to be diseased without a tuberculin test to a cow so badly affected that a calf of which she became the mother, a little less than a year before her blood was used for guinea-pig injections, was born affected with tuberculosis contracted from antepartum exposure to her tuberculous body.

The total number of guinea-pigs injected was 104. Of these 16 died within a few days after the injection and no doubt as a result of it, 3 died of intercurrent affections, but not until a sufficient period of time had passed for lesions of tuberculosis to become clearly manifest, and 85 guinea-pigs lived until they were killed after a lapse of from seven and one-half to eleven weeks, or an average for all of seventy days after they were injected. The 3 guinea-pigs that died of intercurrent affections showed no lesions of tuberculosis on postmortem examination, and 84 of the 85 guinea-pigs that lived until they were killed showed no lesions of any kind on autopsy. One guinea-pig of the 85 showed lesions very slightly resembling tuberculosis, but these were proved by microscopic examinations and guinea-pig inoculation tests to be free from tubercle bacilli.

A detailed record of the cattle and guinea-pigs used in our tests follows:

RECORD OF TUBERCULOUS CATTLE AND GUINEA-PIGS

CATTLE, LOT 1

Bull 393, general condition very good, affected with tuberculosis a year or more, was killed and examined postmortem on April 8, 1909. The autopsy revealed only one small tuberculous lesion located in one of the superficial inguinal glands.

On Feb. 5, 1909, two guinea-pigs, Nos. 2891 and 2892, received each an intraabdominal injection of 2 c.c. of blood from the bull. One guinea-pig, No. 2891, died on Feb. 14, 1909, affected with invagination of the bowel. The other guineapig remained healthy until April 13, 1909 (sixty-seven days after injection), when it was killed and found on postmortem examination to be free from lesions of disease. 
Cow 533 , general condition poor, affected with tuberculosis two years or more, was killed and examined postmortem on April 24, 1909. The autopsy revealed the following conditions; the principal lobe of the right lung contained a cavity about three inches in diameter, partly filled with pasty, necrotic, tuberculous material. This cavity was in direct communication with a large bronchial tube, which contained a considerable amount of material discharged from the cavity. Sprinkled throughout the lungs generally, a number of smaller tuberculous foci, in a completely broken-down condition, were found. The mediastinal and mesenteric lymph glands and the liver were sprinkled with tuberculous foci, some of which were as much as one-half inch in diameter.

Prior to the cow's death her feces were examined microscopically on nine different days and on six of these days were found to contain tubercle bacilli.

On Feb. 3, 1909, two guinea-pigs, Nos, 2859 and 2860, received each an intraabdominal injection of 3 c.c. of blood of the cow. The guinea-pigs remained healthy until April 13, 1909 (sixty-nine days after injection), when they were killed and found on postmortem examination to be free from lesions of disease.

Cow 549, general condition poor, affected with tuberculosis several years, on March 27,1908 , gave birth to a calf affected with congenital tuberculosis. The cow was killed on April 8, 1909, and on autopsy was found to be affected with adranced, generalized tuberculosis. The lungs contained lesions varying from quite recent tuberculous disease to large tuberculous cavities that had discharged most of their contents through the bronchial tubes.

No tests were made relative to the infectious character of the feces before death.

On Feb. 3, 1909, two guinea-pigs, Nos. 2863 and 2864, received each an intraabdominal injection of 3 c.c. of blood from the cow. The guinea-pigs were killed on April 13, 1909 (sixty-nine days after injection), and on postmortem examination were found to be free from lesions of disease.

Cow 552, general condition poor, affected with tuberculosis several years, was killed on April 1, 1909. The autopsy revealed a fairly generalized tuberculosis with lesions of greater or lesser magnitude in the Iungs and in the pharyngeal, bronchial and mesenteric lymph glands.

Prior to the cow's death her feces were examined microscopically on ten different days, and on six of these days were found to contain tubercle bacilli.

Hogs that were fed feces from the cow contracted tuberculosis, and guinea-pigs inoculated subcutaneously with small masses of her feces likewise contracted tuberculosis.

Guinea-pigs were injected intra-abdominally with blood from this cow as follows:
Jan. 25, 1909, Guinea-pig 2785 received 5 c.c. of blood.
Jan. 25, 1909, Guinea-pig 2786 received 5 c.c. of blood.
Jan. 25, 1909, Guinea-pig 2783 received 2.5 c.c. of blood.
Jan. 25, 1909, Guinea-pig 2784 received 2.5 c.c. of blood.
Jan. 25, 1909, Guinea-pig 2781 received 1 c.c. of blood.
Jan. 25, 1909, Guinea-pig 2782 received 1 c.c. of blood.
Feb. 3, 1909, Guinea-pig 2861 received 3 c.c. of blood.
Feb. 3, 1909, Guinea-pig 2862 received 3 c.c. of blood.
Feb. 5, 1909, Guinea-pig 2889 received 2.5 c.c. of blood.
Feb. 5, 1909, Guinea-pig 2890 received 2.5 c.c. of blood.

Guinea-pigs 2786 and 2890 died prematurely as a result of the blood injections, and the remaining eight guinea-pigs were killed on the following dates and on autopsy were found to be free from lesions of disease.

Guinea-pigs 2781 and 2782, killed March 27, 1909 (sixty-one days after in. jection). 
Guinea-pigs 2783 and 2784, killed April 13, 1909 (seventy-eight days after injection).

Guinea-pig 2785, killed April 12, 1909 (seventy-eight days after injection).

Guinea-pig 2889, killed April 13, 1909 (sixty-seven days after injection).

Guinea-pigs 2861 and 2862, killed April 13, 1909 (sixty-nine days after injection).

CATTLE, LOT 2

Cow 511 , general condition poor, has been affected with tuberculosis eighteen months or more. Microscopic examinations of the feces on seven different days revealed tubercle bacilli on three days. A hog fed with feces from the cow contracted tuberculosis.

On Feb. 1, 1909, two guinea-pigs, Nos. 2829 and 2830, received each an intraabdominal injection of 3 c.c. of blood from the cow. The guinea-pigs remained healthy until April 13, 1909 (seventy-one days after injection), when they were killed and found on autopsy to be free from lesions of disease.

Cow 537, general condition fairly good, has been affected with tuberculosis more than two years. Microscopic examinations of the feces on fifteen days revealed tubercle bacilli on eleven days. Guinea-pigs inoculated with small masses of feces contracted tuberculosis.

Guinea-pigs were injected, intra-abdominally, with blood from the cow as follows:

Feb. 4, 1909, Guinea-pig 2871 received 3 c.c. of blood.

Feb. 4, 1909, Guinea-pig 2872 received 3 c.c. of blood.

Feb. 19, 1909, Guinea-pig 3062 received 3 c.c. of blood.

Feb. 19, 1909, Guinea-pig 3063 received 3 c.c. of blood.

Guinea-pig 2872 died prematurely as a result of the injection.

Guinea-pig 2871 was killed on April 13, 1909 (sixty-eight days after injection), and on autopsy was found to be free from lesions of disease. Guinea-pigs 3062 and 3063 were killed on April 13, 1909 (fifty-three days after injection), and on autopsy were found to be free from lesions of disease.

Cow $\tilde{3} 38$, general condition very poor, has been affected with tuberculosis two years or longer. Microscopic examinations of feces on eleven different days revealed tubercle bacilli on nine days. Guinea-pigs inoculated with small masses of feces, and hogs fed feces of this cow, contracted tuberculosis.

On Feb. 3, 1909, two guinea-pigs, Nos. 2851 and 2852, received each an intraabdominal injection of 3 c.c. of blood from the cow. On April 13, 1909 (sixty-nine days after the injection), the guinea-pigs were killed and on autopsy found to be free from lesions of disease.

Cow 555, general condition fairly good, has been affected with tuberculosis more than two years. Microscopic examinations of feces on five different days revealed tubercle bacilli on two days. A hog fed feces from the cow contracted tuberculosis.

On Jan. 30, 1909, two guinea-pigs, Nos. 2811 and 2812, received each an intraabdominal injection of 3 c.e. of blood from the cow. Guinea-pig 2811 died prematurely as a result of the injection. Guinea-pig 2812 was killed on April 12, 1909 (serenty-two days after injection) and on postmortem examination was found to be free from lesions of disease.

Cow $56 \%$, general condition good, has been affected with tuberculosis at least two and a half years. Microscopic examinations of feces on ten different days revealed tubercle bacilli on five days. A hog fed with feces from the cow contracted tuberculosis.

On Feb. 4, 1909, two guinea-pigs, Nos. 2869 and 2870, received each an intraabdominal injection of 3 c.c. of blood from the cow. The guinea-pigs were killed 
on April 12, 1909 (sixty-seven days after injection), and on autopsy were found to be free from lesions of disease.

Cow 6$\}_{f} 6$, general condition fair, has been affected with tuberculosis for some time, but it is not known just how long. Microscopic examinations of feces on two different days revealed tubercle bacilli on one day. Guinea-pigs inoculated with her feces contracted tuberculosis.

On Feb. 2, 1909, two guinea-pigs, Nos. 2847 and 2848, received each an intraabdominal injection of 3 c.c. of blood from the cow. The guinea-pigs were killed on April 13, 1909 (seventy days after injection), and on autopsy were found to be free from lesions of disease.

\section{GATTLE, LOT 3}

Cow 503, general condition good, has been affected with tuberculosis at least two and a half years. Microscopic examinations of feces on two days revealed tubercle bacilli on one day.

On Jan. 30, 1909, two guinea-pigs, Nos. 2805 and 2806, received each an intra. abdominal injection of 3 c.c. of blood from the cow. The guinea-pigs were killed on April 30, 1909 (seventy-three days after injection), and on autopsy were found to be free from lesions of disease.

Cow 510, general condition fairly good, has been affected with tuberculosis about three years. Microscopic examinations of feces on three days revealed tubercle bacilli on two days.

Guinea-pigs injected intra-abdominally with blood from the cow as follows:

$$
\begin{aligned}
& \text { Jan. 29, 1909, Guinea-pig } 2791 \text { received } 5 \text { c.c. } \\
& \text { Jan. 29, 1909, Guinea-pig } 2792 \text { received } 5 \text { c.c. } \\
& \text { Feb. 4, 1909, Guinea-pig } 2881 \text { received } 3 \text { c.c. } \\
& \text { Feb. 4, 1909, Guinea-pig } 2882 \text { received } 3 \text { c.c. }
\end{aligned}
$$

Guinea-pigs 2791 and 2792 died prematurely as a result of the injections. Guinea-pigs 2881 and 2882 were killed on April 13, 1909 (sixty-eight days after injection), and on autopsy were found to be free from lesions of disease.

Cow 512, general condition good, has been affected with tuberculosis eighteen months or longer. Microscopic examinations of feces on six different days revealed tubercle bacilli on four days.

On Feb. 11, 1909, two guinea-pigs, Nos. 2823 and 2824, received each an intraabdominal injection of 3 c.c. of blood from the cow. The guinea-pigs were killed on April 12, 1909 (seventy days after injection), and on autopsy were found to be free from lesions of disease.

Cow 513, general condition fairly good, but with greatly enlarged throat glands, has been affected with tuberculosis eighteen months or Ionger. Microscopic examinations of feces on four different days revealed tubercle bacilli on two days.

On Feb. 2, 1909, two guinea-pigs, Nos. 2835 and 2836, received each an intraabdominal injection of 3 c.c. of blood from the cow. Guinea-pig 2835 died prematurely as a result of the injection. Guinea-pig 2836 was killed on April 12, 1909 (sixty-nine days after injection), and on autopsy was found to be free from lesions of disease.

Cow 51/, general condition poor, has been affected with tuberculosis about three years. Microscopic examinations of feces on three different days revealed tubercle bacilli on one day.

On Jan. 30, 1909, two guinea-pigs, Nos. 2815 and 2816 , received each an intraabdominal injection of 3 c.c. of blood from the cow. Guinea-pig 2816 died of an intercurrent affection on March 3, 1909 (thirty-two days after injection), and on autopsy was found to be free from lesions of tuberculosis. Guinea-pig 2815 was killed on April 12, 1909 (seventy-two days after injection), and on autopsy was found to be free from lesions of disease. 
Cow 515, general condition fairly good, has been affected with tuberculosis eighteen months or longer. Microscopic examinations of feces on seven different days revealed tubercle bacilli on four days.

On Feb. 1, 1909, two guinea-pigs, Nos. 2821 and 2822, received each an intraabdominal injection of 3 c.c. of blood from the cow. The guinea-pigs were killed on April 12, 1909 (seventy days after injection), and on autopsy were found to be free from lesions of disease.

Cow 516, general condition fairly good, has been affected with tuberculosis eighteen months or longer. Microscopic examinations of feces on seven different days revealed tubercle bacilli on four days.

On Feb. 1, 1909, two guinea-pigs, Nos. 2831 and 2832, received each an intraabdominal injection of 3 c.c. of blood from the cow. The guinea-pigs were killed on April 13, 1909 (seventy-one days after injection), and on autopsy were found to be free from lesions of disease.

Cov 536, general condition poor, has been affected with tuberculosis two years or longer. Microscopic examinations of feces on eleven different days revealed tuberele bacilli on eight days.

On Feb. 3, 1909, two guinea-pigs, Nos. 2865 and 2866, received each an intraabdominal injection of 3 c.c. of blood from the cow. The guinea-pigs were killed on April 13, 1909 (sixty-nine days after injection), and on autopsy were found to be free from lesions of disease.

Covo 561, general condition fairly good, has been affected with tuberculosis two vears or longer. Microscopic examinations of feces on nine different days revealed tubercle bacilli on three days.

On Feb. 1, 1909, two guinea-pigs, Nos. 2833 and 2834, received each an injection of 3 c.c. of blood from the cow. The guinea-pigs were killed on April 12, 1909 (seventy days after the injection), and on autopsy were found to be free from lesions of disease.

Cono 553, general condition fairly good, has been affected with tuberculosis two years or longer. Microscopic examinations of feces on three different davs revealed tubercle bacilli on every day.

On Feb. 1, 1909, two guinea-pigs, Nos. 2827 and 2828 , received each an intraabdominal injection of 3 c.c. of blood from the cow. The guinea-pigs were killed on April 13, 1909 (seventy-one days after the injection), and on autopsy were found to be free from lesions of disease.

Cow 620, general condition good, has been affected with tuberculosis a year or longer. Microscopic examinations of feces on four different days revealed tubercle bacilli on one day.

On Feb. 3, 1909, two guinea-pigs, Nos. 2855 and 2856, received each an intraabdominal injection of 3 c.c. of blood from the cow. The guinea-pigs were killed on April 13, 1909 (sixty-nine days after the injection), and on autopsy were found to be free from lesions of disease.

Cow 629 , general condition fair, has been affected with tuberculosis at least one year. Microscopic examinations of feces on five different days revealed tubercle bacilli on two days.

On Feb. 3, 1909, two guinea-pigs, Nos. 2857 and 2858, received each an intraabdominal injection of 3 c.c. of blood from the cow. The guinea-pigs were killed on April 13 (sixty-nine days after the injection), and on autopsy were found to be free from lesions of disease.

Cow 631, general condition fair, has been affected with tuberculosis at least one year. Microscopic examinations of feces on four different days revealed tubercle bacilli on one day.

On Feb. 4, 1909, two guinea-pigs, Nos. 2877 and 2878, received each an intra. abdominal injection of 3 c.c. of blood from the cow. The guinea-pigs were killed 
on April 13, 1909 (sixty-eight days after the injection), and on autopsy were found to be free from lesions of disease.

Bull 635, general condition good, has been affected with tuberculosis over two years. Microscopic examinations of feces on three different days revealed tubercle bacilli on one day.

On Jan. 20, 1909, two guinea-pigs, Nos. 2817 and 2818 , received each an intraabdominal injection of 3 c.c. of blood from the bull. Guinea-pig 2818 died prematurely as a result of the injection. Guinea-pig 2817 was killed on April 12, 1909 (seventy-two days after the injection), and on autopsy was found to be free from lesions of disease.

Covo 636, general condition fairly good, has been affected with tuberculosis at least one year. Microscopic examinations of feces on five different days revealed tubercle bacilli on two days.

On Jan. 30, 1909, two guinea-pigs, Nos. 2803 and 2804, received each an intraabulominal injection of 3 c.c. of blood from the cow. The guinea-pigs were killed on April 12, 1909 (serenty-two days after the injection), and on autopsy were found to be free from lesions of disease.

Cow 638, general condition fairly good, has been affected with tuberculosis over two vears. Microscopic examinations of feces on four different days revealed tubercle bacilli on all four days.

Guinea-pigs were given intra-abdominal injections of the blood of this cow as follows:

Jan. 29, 1909, Guinea-pig 2795 received 5 c.c.

Jan. 29, 1909, Guinea-pig 2796 received 5 c.c.

Feb. 4, 1909, Guinea-pig 2879 received 3 c.c.

Feb. 4, 1909, Guinea-pig 2880 received 3 c.c.

Guinea-pig 2795 died prematurely as a result of the injection. Guinea-pig 2796 was killed on April 12, 1909 (seventy-three days after the injection), and on autopsy was found to be free from lesions of disease. Guinea-pigs 2879 and 2880 were killed on April 12, 1909 (sixty-seven days after injection), and on autopsy were found to be free from lesions of disease.

Cow 699, general condition good, has been affected with tuberculosis at least one year. Microscopic examinations of feces on four different days revealed tubercle bacilli on one day.

Guinea-pigs were given intra-abdominal injections of the blood of this cow as follows:

$$
\begin{aligned}
& \text { Jan. 29, 1909, Guinea-pig } 2801 \text { received } 5 \text { c.c. } \\
& \text { Jan. 29, 1909, Guinea-pig } 2802 \text { received } 5 \text { c.c. } \\
& \text { Feb. 5, 1909, Guinea-pig } 2887 \text { received } 3 \text { c.c. } \\
& \text { Feb. 5, 1909, Guinea-pig } 2888 \text { received } 3 \text { c.c. }
\end{aligned}
$$

Guinea-pig 2801 died prematurely as a result of the injection. Guinea-pig 2802 was killed April 12, 1909 (seventy-three days after the injection), and on autopsy several necrotic foci were found in the liver and spleen. The lesions were not at all like the conditions caused by the tubercle bacillus and microscopic examinations failed to reveal tubercle bacilli. Some of the abnormal tissue was used to make subinoculations into guinea-pigs; the subinoculated guinea-pigs failed to show tuberculosis.

Guinea-pigs 2887 and 2888 were killed on April 13, 1909 (sixty-seven days after the injection), and on autopsy were found to be free from lesions of disease.

Cow 640 , general condition good, has been affected with tuberculosis over two years. Microscopic examinations of feces on three different days revealed tubercle bacilli on all three days.

On Jan. 29, 1909, two guinea-pigs, Nos. 2799 and 2800 , received each an intraabdominal injection of 5 c.c. of blood from the cow. The guinea-pigs were killed 
on April 12, 1909 (seventy-three days after the injection), and on autopsy were found to be free from lesions of disease.

Cow 642, general condition good, has been affected with tuberculosis at least one year. Microscopic examinations of feces on three different days revealed tubercle bacilli on two days. lows:

Guinea-pigs were injected intra-abdominally with blood from this cow as fol-

Jan. 29, 1909, Guinea-pig 2793 received 5 c.c.

Jan. 29, 1909, Guinea-pig 2794 received 5 c.c.

Feb. 5, 1909, Guinea-pig 2885 received 3 c.c.

Feb. 5, 1909, Guinea-pig 2886 received 3 c.c.

Guinea-pig 2794 died prematurely as the result of the injection. Guinea-pig 2886 died on March 5, 1909 (twenty-eight days after the injection), of an intertercurrent affection; the autopsy revealed no lesions resembling tuberculosis. Guinea-pig 2793 was killed on April 13, 1909 (seventy-three days after injection), and on autopsy was found to be free from lesions of disease. Guinea-pig 2885 was killed on April 13, 1909 (sixty-seven days after injection), and on autopsy was found to be free from lesions of disease.

\section{CATTYE, LOT 4}

Cow 479 , general condition fair, but with greatly enlarged throat glands, has been affected with tuberculosis about three years.

On Feb. 4, 1909, two guinea-pigs, Nos. 2873 and 2874 , received each an intraabdominal injection of 3 c.c. of her blood. The guinea-pigs were killed on April 13, 1909 (sixty-eight days after injection), and on autopsy were found to be free from lesions of disease.

Bull 508, general condition good, has been affected with tuberculosis about two and a half years.

On Feb. 4, 1909, two guinea-pigs, Nos. 2867 and 2868, received each an intraabdominal injection of 3 c.e. of his blood. The guinea-pigs were killed on April 12, 1909 (sixty-seven days after injection), and on autopsy were found to be free from lesions of disease.

Cow 517, general condition good, has been affected with tuberculosis about two and a half years.

On Feb. 1, 1909, two guinea-pigs, Nos. 2825 and 2826, received each an intraabdominal injection of 3 c.e. of her blood. Guinea-pig 2825 died of an intercurrent affection on April 5, 1909 (sixty-three days after injection), and on autopsy was found to be free from lesions of tuberculosis. Guinea-pig 2826 was killed April 13, 1909 (seventy-one days after injection), and on autopsy was found to be free from lesions of disease.

Cow 569, general condition fair, has been affected with tuberculosis about two years.

On Feb. 1, 1909, two guinea-pigs, Nos. 2819 and 2820 , received each an intraabdominal injection of 3 c.c. of her blood. The guinea-pigs were killed April 12, 1909 (seventy days after injection), and on autopsy were found to be free from lesions of disease.

Cow 630 , general condition fair, has been affected with tuberculosis about one year.

On Feb. 3, 1909, two guinea-pigs, Nos. 2853 and 2854 , received each an intraabdominal injection of 3 c.c. of her blood. The guinea-pigs were killed April 13, 1909 (sixty-nine days after the injection), and on autopsy were found to be free from lesions of disease.

Cow 692, general condition good, has been affected with tuberculosis at least one year. 
On Jan. 30, 1909, two guinea-pigs, Nos. 2807 and 2808, received each an intraabdominal injection of 3 c.c. of her blood. Guinea-pig 2808 died prematurely as a result of the injection. Guinea-pig 2807 was killed on April 13, 1909 (seventy-two days after the injection), and on autopsy was found to be free from lesions of disease.

Cow 634 , general condition fair, has been affected with tuberculosis at least one year.

On Jan. 29, 1909, two guinea-pigs, Nos. 2787 and 2788, received each an intraabdominal injection of 5 c.c. of her blood. The guinea-pigs were killed on April 12,1909 (seventy-three days after the injection), and on autopsy were found to be free from lesions of disease.

Cow 641 , general condition fair, has been affected with tuberculosis over two years.

On Jan. 29, 1909, two guinea-pigs, Nos. 2797 and 2798, received each an intraabdominal injection of 5 c.c. of the blood of the cow, and on Feb. 4, 1909, two guinea-pigs, Nos. 2875 and 2876 , received each a similar injection of 3 c.c. of blood. Guinea-pig 2798 died prematurely as a result of the injection. Guinea-pig 2797 was killed on April 12, 1909 (seventy-five days after the injection), and guineapigs 2875 and 2876 were killed on April 12, 1909 (sixty-seven days after the injection). The three guinea-pigs were found to be free from lesions of disease.

Cow 644, general condition fair, has been affected with tuberculosis four months or more.

On Feb. 2, 1909, two guinea-pigs, Nos. 2849 and 2850 , received each an intraabdominal injection of 3 c.c. of blood of the cow. The guinea-pigs were killed on April 13, 1909 (seventy days after the injection), and on autopsy were found to be free from lesions of disease.

Cow 645, general condition good, has been affected with tuberculosis three months or more.

On Feb. 2, 1909, two guinea-pigs, Nos, 2845 and 2846, received each an intraabdominal injection of 3 c.c. of her blood. Guinea-pig 2845 died prematurely as a result of the injection. Guinea-pig 2846 was killed on April 13, 1909 (seventy days after the injection), and on autopsy was found to be free from lesions of disease.

Cow 648 , general condition good, has been affected with tuberculosis an unknown period of time. She was brought to the experiment station only shortly before her blood was used for guinea-pig injections and reacted with tuberculin.

On Feb. 2, 1909, two guinea-pigs, Nos. 2837 and 2838 , received each an intraabdominal injection of 3 c.c. of her blood. The guinea-pigs were killed on April 12, 1909 (sixty-nine days after the injection), and on autopsy were found to be free from lesions of disease.

Cow $65 \%$, general condition fair, has been affected with tuberculosis an unknown period of time. She was brought to the experiment station only shortly before her blood was used for guinea-pig injections, and reacted with tuberculin.

On Feb. 2, 1909, two guinea-pigs, Nos. 2841 and 2842, received each an intraabdominal injection of 3 c.c. of her blood. The guinea-pigs were killed on April 12,1909 (sixty-nine days after the injection), and on autopsy were found to be free from lesions of disease.

The 42 cattle included in the records are all those that were arailable for this investigation among the tuberculous cattle kept for various purposes at the Bureau of Animal Industry Experiment Station. The general condition of the cattle is briefiy defined as good, fairly good. fair or poor, and, as these terms are used somewhat arbitrarily, it is desirable to specify more precisely the ideas they are intended to convey. 
The word "good" is used in connection with cattle that are really in excellent, risible, physical condition and in which no one would suspect disease; the words "fairly good" are used to mean that condition commonly found among dairy cows of the better class; "fair" is used to designate a condition which the average dairyman regards as satisfactory, and the word "poor" is used to qualify all cattle that are thin or that show visible symptoms of disease.

Among the 42 cattle 27 , or 64.25 per cent., were shown by microscopic examinations to be discharging tubercle bacilli from their bowels, in most instances intermittently, and the infectious character of the feces of 7 of the 42 cattle, or $16 \% 3$ per cent., was proved by animal experiments; that is, feeding and inoculation tests.

The two facts, that 27 of the cattle were shown by microscopic tests to be expelling tubercle bacilli per rectum and that only 7 were proved by animal experiments to be passing infected feces, must not be taken as being in any sense contradictory, as the feces of only a sufficient number of tuberculous cattle were tested by animal feeding and inoculation experiments to prove conclusively that the acid-fast bacilli found on microscopic examinations in the feces of tuberculous cattle are certainly live, virulent tubercle bacilli.

Relative to the expulsion of tubercle bacilli from the bowels of tuberculous cattle, we wish to say that all the evidence we have indicates that the bacilli have their origin in the lungs and throat, from which regions they are coughed up, swallowed, and passed through and out of the intestinal canal without appreciable loss of pathogenic virulence. That a large proportion of the tubercle bacilli swallowed by cattle really pass throngh their bodies and out per rectum, without a determinable loss of virulence, was experimentally shown in some of our earlier work. 2 We have absolutely no reason to believe that tubercle bacilli enter the intestinal canal from the lymph radicles or blood capillaries, or by any complex and mysterious system of transportation from lesions of all descriptions and kinds in any or every portion of the body. It is our conviction that, unless an open tuberculosis is in more or less direct communication with the intestinal canal or there is a tuberculous disease of the intestine itself, which latter is rare among cattle, no tubercle bacilli will be expelled with the feces.

If tuberculosis in all its forms was a bacteriemia, the expulsion of tubercle bacilli from the bowels of all tuberculous individuals, as well

2. Bull. 88 and 99, Bureau of Animal Industry. 
as with their urine, saliva, milk and other bodily secretions, would follow as a natural consequence. Those who have carefully studied the secretions from the uninvolved organs of tuberculous subjects know how rarely tubercle bacilli are detected in them, even with the application of the most delicate tests.

When we consider cattle like Nos. 533, 549 and 552, three of the four animals of which autopsy records are given, and note that they were so badly diseased that they would have been condemned on superficial examination as wholly unfit for use as food under the existing meat inspection regulations, the absence of tubercle bacilli from their blood may be regarded as a sufficient reason for assuming that the possible occurrence of tubercle bacilli in the blood of tuberculous animals will almost invariably be associated with pathologic conditions of a very marked character, or that the tubercle bacilli will be present in extremely small numbers and will speedily be filtered out of the blood stream. Cow 533 had been affected with tuberculosis two years or longer, was in poor condition as a result of the disease, and on autopsy was found to have an extensive, open tuberculosis of the lung, and lesions of tuberculosis in the liver, and in both the thoracic and abdominal lymph glands. Cow 549 was, if anything, even more severely and extensively affected, and less than a year before her blood was injected into guineapigs had given birth to a congenitally tuberculous calf. Cow 552 was also affected with generalized, advanced, open tuberculosis, and prior in the use of her blood for guinea-pig injections was found to be passing large numbers of tubercle bacilli from her bowels, which were proved by feeding tests to be virulent for hogs, and by inoculation tests to be virulent for guinea-pigs. With the blood obtained from these 3 cows 14 guinea-pigs were injected, 12 of which lived two months or more afterward, until they were intentionally killed, when they were found on postmortem examination to be wholly free from Iesions of disease of any kind.

The possibility exists that tubercle bacilli introduced into the stomach and intestine by swallowing may be taken up by the lymph radicles, passed along the lymph channels and emptied through the great lymph ducts into the venous circulation. The investigations of Nicolas and Descos, Ravenel, Calmette and Guerin, Schlossman and Engle, and others speak for this, but such tubercle bacilli will not be very numerous and will no doubt be filtered out of the blood as soon as it reaches the lung through the heart and pulmonary arteries, to which it passes directly after it has received the lymph stream. 


\section{CONCLUSIONS}

We failed utterly to find tubercle bacilli in the blood of tuberculous cattle which we examined microscopically in accordance with the method described and used by Dr. Rosenberger.

The negative results of our microscopic examinations are confirmed by the negative results obtained with 88 guinea-pigs, each of which received an intra-abdominal injection of blood from a tuberculous cow or bull.

As the number of cattle from which blood was injected into the 88 guinea-pigs is 42 , and as these cattle represented practically all stages of tuberculosis, from mildly affected, recent cases to old and completely generalized cases, we feel that our work shows beyond the remotest doubt that tuberculosis is not to be classified, in any sense of the word, as a bacteriemia.

\section{SUPPLEMENT}

In the foregoing article we made the statement: "The possibility exists that the intraperitoneal injection of from 3 to 5 c.c. of fresh, warm blood from tuberculous cattle induces an immunity in guinea-pigs to the tubercle bacilli the blood may contain. Though this view is purely hypothetical and we know of nothing to sustain it, we have undertaken an investigation to prove or disprove it, and will append the results to this article if they are ready before it goes to the printer." The results of the additional investigation are now ready and are given in this supplement.

On April 24, 1909, blood and tuberculous material were obtained from Cow 533 (see record 8) for a number of guinea-pig injections. The primary object of the injections was to prove that the blood of a tuberculous cow, when introduced into the peritoneal cavity of a guinea-pig, has no retarding influence on the development of tuberculosis from tubercle bacilli that may be present in such blood.

Cow 533 was first bled from the jugular vein and then at once killed. As soon as she was dead a tuberculous mediastinal gland was removed from her body and $500 \mathrm{mg}$. of it emulsified with 2 c.c. of sterile, normal salt solution. Cover-glasses of this emulsion, stained with carbolfuchsin and decolorized with 20 per cent. sulphuric acid, on microscopic examination revealed, on an average, two tubercle bacilli each. The emulsion was mixed with an additional quantity of sterile, normal salt solution, so that each cubic centimeter of the dilution represented a strength equal to one drop of the original emulsion.

The blood obtained from the cow prior to her death, and the diluted emulsion made with the tuberculous mediastinal gland from her body, were used to inject seven groups of guinea-pigs, the records of which follow.

Group 1.-On April 24, 1909, eight guinea-pigs, Nos. 3626 to 3633 inclusive, received each an intra-abdominal injection of 3 c.c. of freshly drawn, warm blood. On May 6, 1909, guinea-pig 3627 died affected with congestion of the lungs. On autopsy no lesions of tuberculosis were found. On May 27 and 28, 1909, guineapigs Nos. 3626, 3628, 3629, 3630, 3631, 3632 and 3633 were killed and examined postmortem. No lesions of tuberculosis or other disease were found.

Group 2.-On April 24, 1909, eight guinea-pigs, Nos. 3642 to 3649 inclusive, received each an intra-abdominal injection of 3 c.c. of freshly drawn blood followed as quickly as possible by an intra-abdominal injection of 0.5 c.c. of tuberculous 
emulsion. On April 30, 1909, guinea-pig 3642 died affected with inflammation of the large bowel. On May 27, 1909, guinea-pigs 3643, 3644, 3645, 3646, 3647, 3648 and 3649 were killed and examined postmortem. Every one of the seven guineapigs was found to be affected with generalized tuberculosis of the abdominal and thoracic organs.

Group 8.-On April 24, 1909, eight guinea-pigs, Nos. 3634 to 364 I inclusive, received each an intra-abdominal injection of freshly drawn, warm blood, followed as soon as possible by a subcutaneous injection into the right thigh of 0.5 c.c. of emulsion. On May 27, 1909, guinea-pigs 3636 and 3637, and on May 28, 1909, guinea-pigs $3634,3635,3638,3639,3640$ and 3641 were killed and examined postmortem. The eight guinea-pigs all showed more or less extensive lesions of tuberculosis at the seat of the subcutaneous injection, tuberculosis of the adjacent, superficial inguinal gland, tuberculosis of the pelvic, lumbar and gastrohepatic glands and a sprinkling of tuberculous foci in the liver and spleen.

Group 4.-On April 24, 1909, eight guinea-pigs, Nos. 3658 to 3665 inclusive, received each an intra-abdominal injection of 3 c.c. of a mixture of defibrinated blood and tuberculous emulsion. Each 3 c.c. of this mixture was equivalent to 0.5 c.c. of the earlier described, diluted tuberculous emulsion. The mixture was two hours old at the time it was injected into the guinea-pigs. On April 25, 1909, guinea-pigs 3659,3660 and 3661 died. The postmortem examination showed no lesions excepting a congested condition of the lungs and a quantity of unabsorbed blood in the peritoneal cavity. On May 12, 1909, guinea-pig 3662 died affected with congestion of the lungs; no lesions of tuberculosis were found. On May 27, 1909, guinea-pigs $3658,3663,3664$ and 3665 were killed and examined postmortem. Three of the guinea-pigs were affected with completely generalized tuberculosis of the abdominal and thoracic organs and one, No. 3665, with generalized tubereulosis of the abdominal organs only.

Group 5.-On April 26, 1909, eight guinea-pigs, Nos. 3670 to 3677 inclusive, received each an intra-abdominal injection of 3 c.c. of the same mixture of blood and tuberculous emulsion used for the guinea-pigs of group 4. The mixture was forty-five hours old at the time it was injected into the guinea-pigs. On May 27, 1909 , guinea-pigs $3670,3671,3672,3673,3674,3675,3676$ and 3677 were killed and examined postmortem. Seven of the guinea-pigs were affected with generalized tuberculosis of the abdominal and thoracic organs and one, No. 3672, with genera]. ized tuberculosis of the abdominal organs only.

Group 6.-On April 24, 1909, four guinea-pigs, Nos. 3650 to 3653 inclusive, received each an intra-abdominal injection of 0.5 c.c. of tuberculous emulsion. On May 6, 1909, guinea-pig 3651 died affected with congestion of the lungs. On May 27 , 1909, guinea-pigs 3650,3652 and 3653 were killed and examined postmortem. Guinea-pig 3650 showed tuberculous lesions of the spleen and omentum only and guinea-pigs 3652 and 3653 showed a fairly generalized tubereulosis of the abdominal and thoracic organs.

Group 7.-On April 24, 1909, four guinea-pigs, Nos. 3654 to 3657 inclusive, received each a subcutaneous injection, right thigh, of 0.5 c.c. of tuberculous emulsion. On May 27, 1909, guinea-pigs 3654, 3655, 3656 and 3657 were killed and examined postmortem. Guinea-pigs 3654 and 3656 showed each a small, tuberculous abscess at the seat of injection, a tuberculous condition of the superficial inguinal gland near the seat of injection and a fairly generalized tuberculosis of the pelvic and abdominal organs. Guinea-pigs 3655 and 3657 showed similar lesions, with the exception of abscesses at the seat of injection.

The guinea-pigs in the seven different groups were injected for the following purposes: Group 1, to serve as checks on the absence or presence of tubercle bacilli 
in the blood of the tuberculous cow that was used for the investigation; Group 2, to show that the intra-abdominal injection of fresh, warm blood from a tuberculous cow can not protect against tubercle bacilli simultaneously introduced into the abdominal cavity; Group 3, to show that the intra-abdominal injections of fresh, warm blood from a tuberculous cow can not protect against tubercle bacilli introduced into other parts of the body than the abdominal cavity; Groups 4 and 5, to show that the blood of tuberculous cows has no special germicidal potency for tubercle bacilli; and Groups 6 and 7 to serve as guides relative to the amount of tuberculous disease to be expected in the bodies of the guinea-pigs that were injected with both blood and emulsion of tuberculous material.

The autopsy records of the guinea-pigs show, in a general way, very little difference between the animals that received only tuberculous emulsion and those that received both blood and emulsion. The guinea-pigs that rceived both blood and emulsion into their abdominal cavities showed numerically more extensive lesions of tuberculosis than the guinea-pigs that received only emulsion into their abdominal cavities. This condition would naturally be expected because the same number of tubercle bacilli contained in 3 c.c. of blood would be more widely separated and in better condition to start a large number of individual lesions than those in 0.5 c.c. of salt solution.

The total number of guinea-pigs injected in this supplemental investigation is 48, of which 8 received blood only, 32 both blood and tuberculous material and $s$ tuberculous material only. Of the 32 that received both blood and tuberculous material and the 8 that received only tuberculous material, 6 guinea-pigs died prematurely and the remaining 34 , when they were killed, thirty to thirtyone days after the injection, were all found to be affected with tuberculosis of a form that would have progressed to death in a short time.

Among the 8 guinea-pigs that received an injection of fresh warm blood without the addition of tuberculous material, 1 died prematurely and the remaining 7 were found on autopsy to be free from lesions of disease. As the cow that supplied the blood for the injections was affected, as her record shows, with extensive, advanced tuberculosis, the 7 guinea-pigs make a strong addition to the 88 parallel guinea-pigs of which the records are given in the article to which this supplement is attached, and hence we have 95 guinea-pigs as the total number that received injections of blood from tuberculous eattle into their peritoneal cavities, the most delicate test for tubercle bacilli available, and survived the injection long enough for tuberculosis to clearly manifest itself. Among this total of 95 guinea-pigs, not one case of tubereulosis developed.

The use of an emulsion of tuberculous tissue from the tuberculous cow that supplied the blood for the supplemental injections was preferred to the use of a pure culture of tubercle bacilli, because it seemed preferable to us to use infectious material and blood in this instance from the same individual case of tuberculosis.

The conclusion we draw from our work is this: If tubercle bacilli ever float in the circulating blood of tuberculous individuals, the occurrence is very rare, so rare indeed, that no form of tuberculosis can reasonably be characterized as a bacteriemia. ${ }^{3}$

Mohlex examined the blood of 8 cattle microscopically and with blood from each of these cattle injected 5 guinea-pigs. The microscopic examinations and injections were made precisely in the manner described by Dr. Rosenberger. No tubercle bacilli were discovered microscopically and not one of the 40 injected

3. An independent investigation relative to the occurrence of tubercle bacilli in the circulating blood of cattle was made at the Federal Bureau of Animal Industry by Dr. John R. Mohler, Chief of the Division of Pathology. 
guinea-pigs contracted tuberculosis. Two of the 8 cattle were in good condition, but were passing tubercle bacilli from their bowels; 2 of the cattle were in poor condition and were passing tubercle bacilli from their bowels; and 4 of the cattle were siaughtered for meat, but on inspection were found to be so extensively affected with tuberculosis that it was necessary to condemn and tank their car. casses under the federal meat inspection regulations.

This additional investigation is especially important because the methods used to discover tubercle bacilli in the blood were identical throughout with those used by Dr. Rosenberger, and which, in Rosenberger's work, invariably gave positive results. 